OPEN ACCESS

Edited by:

Claude Desplan

New York University, United States

Reviewed by:

Thomas Cronin,

University of Maryland, Baltimore

County, United States

Adriana Briscoe,

University of California, Irvine,

United States

${ }^{*}$ Correspondence:

Kentaro Arikawa

arikawa@soken.ac.jp

Received: 24 September 2017 Accepted: 15 November 2017

Published: 29 November 2017

Citation:

Arikawa K, Iwanaga T, Wakakuwa M and Kinoshita M (2017) Unique

Temporal Expression of Triplicated Long-Wavelength Opsins in

Developing Butterfly Eyes.

Front. Neural Circuits 11:96.

doi: 10.3389/fncir.2017.00096

\section{Unique Temporal Expression of Triplicated Long-Wavelength Opsins in Developing Butterfly Eyes}

\author{
Kentaro Arikawa ${ }^{1 \star}$, Tomoyuki Iwanaga ${ }^{2}$, Motohiro Wakakuwa ${ }^{1}$ and Michiyo Kinoshita ${ }^{1}$ \\ ${ }^{1}$ Laboratory of Neuroethology, Department of Evolutionary Studies of Biosystems, Graduate University for Advanced Studies \\ (SOKENDAI), Hayama, Japan, ²Graduate School of Integrated Science, Yokohama City University, Yokohama, Japan
}

Following gene duplication events, the expression patterns of the resulting gene copies can often diverge both spatially and temporally. Here we report on gene duplicates that are expressed in distinct but overlapping patterns, and which exhibit temporally divergent expression. Butterflies have sophisticated color vision and spectrally complex eyes, typically with three types of heterogeneous ommatidia. The eyes of the butterfly Papilio xuthus express two green- and one red-absorbing visual pigment, which came about via gene duplication events, in addition to one ultraviolet (UV)- and one blue-absorbing visual pigment. We localized mRNAs encoding opsins of these visual pigments in developing eye disks throughout the pupal stage. The mRNAs of the UV and blue opsin are expressed early in pupal development (pd), specifying the type of the ommatidium in which they appear. Red sensitive photoreceptors first express a green opsin mRNA, which is replaced later by the red opsin mRNA. Broadband photoreceptors (that coexpress the green and red opsins) first express the green opsin mRNA, later change to red opsin mRNA and finally re-express the green opsin mRNA in addition to the red mRNA. Such a unique temporal and spatial expression pattern of opsin mRNAs may reflect the evolution of visual pigments and provide clues toward understanding how the spectrally complex eyes of butterflies evolved.

Keywords: insect, compound eye, ommatidium, photoreceptor, development, eye disk, visual pigment, rhodopsin

\section{INTRODUCTION}

The structure and function of visual systems vary depending on animals' habitats and foraging strategies. For example, flower-visiting diurnal insects often have spectrally-richer eyes than nocturnal non-flower visitors whose eyes have higher absolute sensitivity instead. What is the proximate cause of creating such evolutionary diversity? How did some eyes become spectrally complex compared to their simpler ancestors?

Compound eyes consist of thousands of ommatidia each housing several photoreceptor cells, with the precise number of photoreceptors differing among species (Figure 1; Friedrich et al., 2011; Wernet et al., 2015). The ommatidia can typically be divided into two or three spectrally distinct types according to the spectral sensitivities of their photoreceptors. Interestingly, species with two ommatidial types in their main retina, such as locusts (Schmeling et al., 2014), leafhoppers (Wakakuwa et al., 2014), and flies (Franceschini et al., 1981; Chou et al., 1999) have eight photoreceptors (R1-8) in each ommatidium. On the other hand, 
the ommatidia of butterflies and bees typically bear nine photoreceptor cells (R1-9), and their main retinas contain three types of ommatidia (Figures 1A,C).

The ninth photoreceptor in butterflies and bees serves to enhance their eyes' spectral sophistication. During development, the eyes of butterflies contain two photoreceptor precursors per ommatidium that express the transcription factor Prospero, which is expressed in the precursor of the R7 photoreceptor in Drosophila melanogaster (Cook et al., 2003). Once expressing Prospero, a subset of Drosophila R7 photoreceptors (dR7s) then stochastically expresses the transcription factor Spineless while others do not, and this binary distinction divides the ommatidia into types (Wernet et al., 2006). Because butterfly ommatidia contain two dR7-like Prospero-positive cells, there are three possible combinations: ON-OFF, OFF-OFF and ON$\mathrm{ON}$, and these Spineless expression patterns indeed correspond precisely with the three types of ommatidia in the Japanese yellow swallowtail butterfly, Papilio xuthus (Figure 1D; Arikawa, 2003; Perry et al., 2016).

Spectral richness of compound eyes is often achieved via visual pigment gene duplication (Briscoe, 2008), which facilitates improved color vision. The ancestral insect eye likely expressed three visual pigment opsins, corresponding to visual pigments absorbing short-wavelength or ultraviolet (UV), middle-wavelength or blue (B), and long-wavelength or green (G; Chang et al., 1996; Townson et al., 1998; Wakakuwa et al., 2005). This presumed ancestral scheme still exists in honeybees, with these visual pigments forming the physiological basis of their UV-B-G trichromacy (von Helversen, 1972). However, butterflies often have more than three opsins: for example, Papilio xuthus has two G opsins (G1 and G2) and one red (R) opsin in addition to the UV and B opsins (Kitamoto et al., 1998), due to repeated gene duplication events (Briscoe, 1999). As a result, the eyes of Papilio xuthus are more spectrally complex, containing six classes of spectral receptor. The $\mathrm{R}$ opsin is expressed in a subset of photoreceptors, which are thus red sensitive (Figure 1). Even with six classes of spectral receptors in the eye, color vision is not necessarily hexachromatic: the wavelength discrimination function indicates that Papilio color vision is UV-B-G-R tetrachromatic (Koshitaka et al., 2008).

Some of the new opsins acquired via gene duplication processes are coexpressed with other opsins within single photoreceptors, enhancing the photoreceptors' spectral variation further. Coexpression of opsins contradicts the classical one cell-one opsin concept, but accumulating evidence indicates that this phenomenon is much more common than had previously been thought, both in invertebrates and vertebrates. These opsin-coexpressing photoreceptors often exist in retinal margins or around the border of distinct retinal regions, suggesting that they may be merely imperfectly differentiated (Röhlich et al., 1994; Makino and Dodd, 1996; Parry and Bowmaker, 2002; Hu et al., 2011). However, recent studies have shown that such photoreceptors occupy specific position in the retinal mosaic, implying functional importance (Mazzoni et al., 2008; Rajkumar et al., 2010; Dalton et al., 2014; Chen et al., 2016; McCulloch et al., 2017). For example, the
Papilio R5-8 (pR5-8) photoreceptors of type II ommatidia (Figure 1D) are broad-band receptors, coexpressing G2 and $\mathrm{R}$ opsins (Arikawa et al., 2003). The green sensitive pR3 and pR4 photoreceptors coexpress G1 and G2 across all but the limited dorsal region of the eye (Kitamoto et al., 1998).

Presently, it is poorly understood how the coexpression of multiple opsins is controlled after ommatidial fate determination, and how such complex eye organization has evolved in butterflies. To investigate these issues, we studied the anatomy of developing compound eyes in Papilio xuthus with particular attention to the expression of opsin mRNAs in photoreceptor precursors.

\section{MATERIALS AND METHODS}

\section{Animals}

We used Japanese yellow swallowtails, Papilio xuthus, from a stock culture derived from individuals captured in Kanagawa, Japan. Larvae were reared on fresh citrus leaves under a light regime of $14 \mathrm{~h}$ light:10 $\mathrm{h}$ dark at $25 \pm 1^{\circ} \mathrm{C}$, which produces non-diapausing pupae. The pupae were kept under the same conditions. Adult females emerge on the 11th day after pupation; the pupal period is 10 days. The pupal period of males is 1 day shorter, and we used only females in this study for simplicity and clarity. We used at least five individuals per each individual stage.

\section{Anatomy}

To determine the stages of eye development, we studied the morphology of eye disks (pupal eye tissue) under a dissecting microscope at $24 \mathrm{~h}$ intervals starting from the day of pupation ( 1 day pupa) to 1 day before adult eclosion (10 day pupa).

For light and electron microscopy, eye disks were prefixed in $2 \%$ glutaraldehyde, $2 \%$ paraformaldehyde in $0.1 \mathrm{M}$ sodium cacodylate buffer $(\mathrm{CB}, \mathrm{pH} 7.4)$ for $2 \mathrm{~h}$ at room temperature and postfixed in $2 \%$ osmium tetroxide in $\mathrm{CB}$ for $2 \mathrm{~h}$ at room temperature. The tissues were then dehydrated with an acetone series and embedded in Epon. Sections of $5 \mu \mathrm{m}$ thickness were stained with Azur-II for light microscopy. Ultrathin sections were stained with uranyl acetate and were observed in Hitachi H-7650 electron microscope. For immunohistochemistry, eye disks were fixed in $4 \%$ paraformaldehyde in $0.1 \mathrm{M}$ sodium phosphate butter ( $\mathrm{pH}$ 7.4) for 2-6 h on ice; see Perry et al. (2016) for the labeling procedure.

\section{RT-PCR Analysis}

The Papilio retina contains five opsin mRNAs each encoding $\underline{\text { Papilio }}$ xuthus ultraviolet-absorbing (PxUV), PxB (blue), PxL1 (green), PxL2 (green) and PxL3 (red) (Kitamoto et al., 1998, 2000). We determined the post-pupation day at which each of these opsin mRNAs became detectable by reverse transcription polymerase chain reaction (RT-PCR). Total RNA was extracted from the eye tissues of the pupa every $24 \mathrm{~h}$ using the RNAgents Total RNA Isolation Kit (Promega), and cDNA was synthesized using oligo-dT primer by reverse 
A

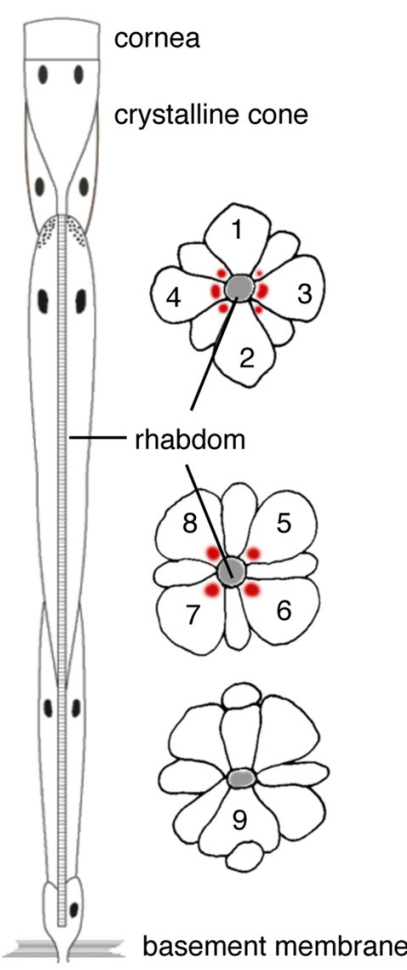

B

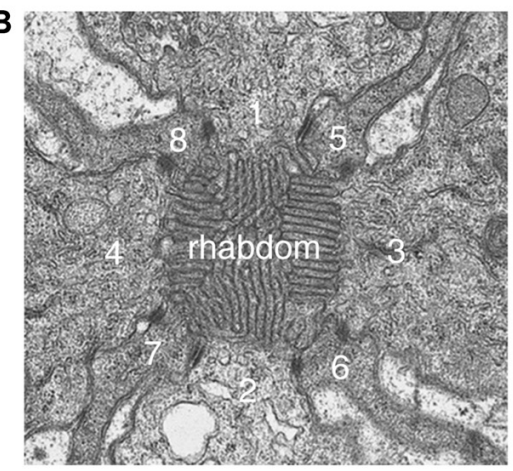

C

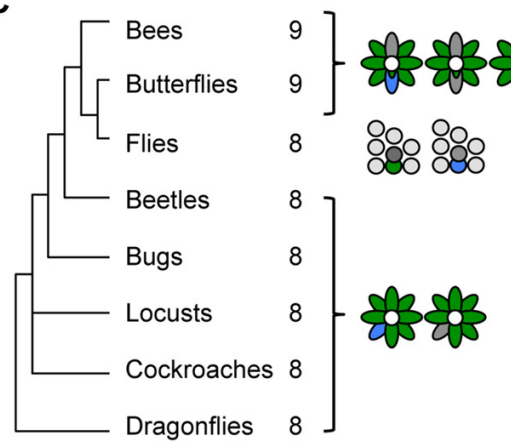

D

\begin{tabular}{|c|c|c|c|c|c|c|c|c|c|}
\hline \multirow{4}{*}{$\begin{array}{r}\text { type of ommatidia: } \\
\text { pigmentation: } \\
\text { fluorescence: } \\
\text { photoreceptors }\end{array}$} & \multicolumn{3}{|c|}{ type I } & \multicolumn{3}{|c|}{ type II } & \multicolumn{3}{|c|}{ type III } \\
\hline & & \multicolumn{3}{|c|}{ red } & \multicolumn{3}{|c|}{ yellow } \\
\hline & \multicolumn{3}{|c|}{ no } & \multicolumn{3}{|c|}{ yes } & \multicolumn{3}{|c|}{ no } \\
\hline & $S(\lambda)$ & Opsin & Ss & $S(\lambda)$ & Opsin & Ss & $S(\lambda)$ & Opsin & Ss \\
\hline pR1 & UV & PxUV & OFF & V & PxUV & OFF & B & $P \times B$ & ON \\
\hline pR2 & B & $\mathrm{P} \times \mathrm{B}$ & ON & V & PxUV & OFF & B & PxB & ON \\
\hline pR3-4 & DG & $P \times G 1+G 2$ & - & SG & $P \times G 1+G 2$ & $2-$ & DG & $\mathrm{PxG} 1+\mathrm{G} 2$ & - \\
\hline pR5-8 & $\mathrm{R}$ & PxR & - & BB & $P \times G 2+R$ & - & DG & PxG2 & - \\
\hline pR9 & $\mathrm{R}$ & $?$ & - & $?$ & $?$ & - & G & $?$ & - \\
\hline
\end{tabular}

FIGURE 1 | Butterfly ommatidia. (A) Diagram of a tiered ommatidium. Nine photoreceptors (1-9) form a phototransductive rhabdom along the central axis. (B) Transverse section of distal rhabdom of adult. (C) Insect phylogeny and photoreceptor number per ommatidium. Right diagrams show typical ommatidial types. (D) Three ommatidial types of Papilio xuthus. Information about pR9 photoreceptors is limited, but see also Figure 5 in Briscoe (2008) for opsins expressed in the pR9 cells of Papilio glaucus. Ss, Spineless; $S(\lambda)$, spectral sensitivity.

transcription. The primers were designed to amplify 200-300 bp fragment of one of the opsin mRNA. The sequences of the primers are as follows: PxUVF (PxUV-Forward), TGAAT TCACA TATAC TGACC CAACG CG; PxUVR (PxUV-Reverse) GGAAA GCTTT CCATT ATTCA CGCCA GTTC; PxBF, AGAAT TCTCC AACGA ACGAT GCAAT CG; PxBR, CGAAA GCTTT CGGAG TCCAT ACAAC AAGC; PxL1F, TGAAT TCAAC GACGA CGAAT GTTTG CG; PxL1R, TAAAA GCTTT TACTA TCGCA GGCTA AC; PxL2F, GGAAT TCCCC TAAGG ATCTG ATACT GC; PxL2R, ACCAA GCTTG GTACA CAGCT TGTTT CATC; PxL3F, TGAAT TCAAC CAACG ATGAC GACTT GG; PxL3R, GATAA GCTTA TCACA CGAGG ATAGT AGGG. We also used primer sets for amplifying cDNA of actin (forward = CAYAC NGTIC CNATH TAYGA RGG; reverse $=$ TCIGC DATNC CNGGR TACAT NGT).

\section{In Situ Hybridization}

The pupal eye tissues were fixed in $4 \%$ paraformaldehyde in $0.1 \mathrm{M}$ buffered sodium phosphate ( $\mathrm{pH} 7.2, \mathrm{~PB})$ for $0.5-2 \mathrm{~h}$ at $25^{\circ} \mathrm{C}$. After dehydration with an ethanol series, they were embedded in paraplast, sectioned at $8 \mu \mathrm{m}$ thickness, mounted on poly-L-lysine-coated slide and dried overnight at $37^{\circ} \mathrm{C}$.
Probes for in situ hybridization were designed to hybridize to about 400 bases of the mRNA in the non-coding region downstream of the C-terminal. The corresponding cDNA region was first subcloned into pGEM-3zf $(+)$ vector, and then digoxigenin (DIG)-labeled cRNA was generated using the DIG-RNA labeling kit (Roche).

The sections were deparafinized and treated with hybridization solution ( $300 \mathrm{mM} \mathrm{NaCl}, 2.5 \mathrm{mM}$ EDTA, $200 \mathrm{mM}$ Tris- $\mathrm{HCl}(\mathrm{pH} 8.0), 50 \%$ formamide, $10 \%$ dextran sulfate, $1 \mathrm{mg} / \mathrm{ml}$ yeast tRNA, $1 \times$ Denhardt's medium), containing $0.5 \mathrm{mg} / \mathrm{ml}$ of the cRNA probe at $45^{\circ} \mathrm{C}$ overnight. After a brief rinse, the sections were incubated in $50 \%$ formamide in $2 \times$ SSC (saline sodium citrate buffer) at $55^{\circ} \mathrm{C}$ for $2 \mathrm{~h}$, and then treated with RNase $(10 \mathrm{mg} / \mathrm{ml})$ at $37^{\circ} \mathrm{C}$ for $1 \mathrm{~h}$. The probes were further visualized by anti-DIG immunocytochemistry.

\section{RESULTS}

\section{Cellular Organization}

The developing compound eye (eye disk) in the day 1 pupa is a sheet of tissue attached to the pupal cuticle by connective tissue (Figure 2A). It expands and detaches from the cuticle by day 4 . The characteristic dome shape of the mature eye is 


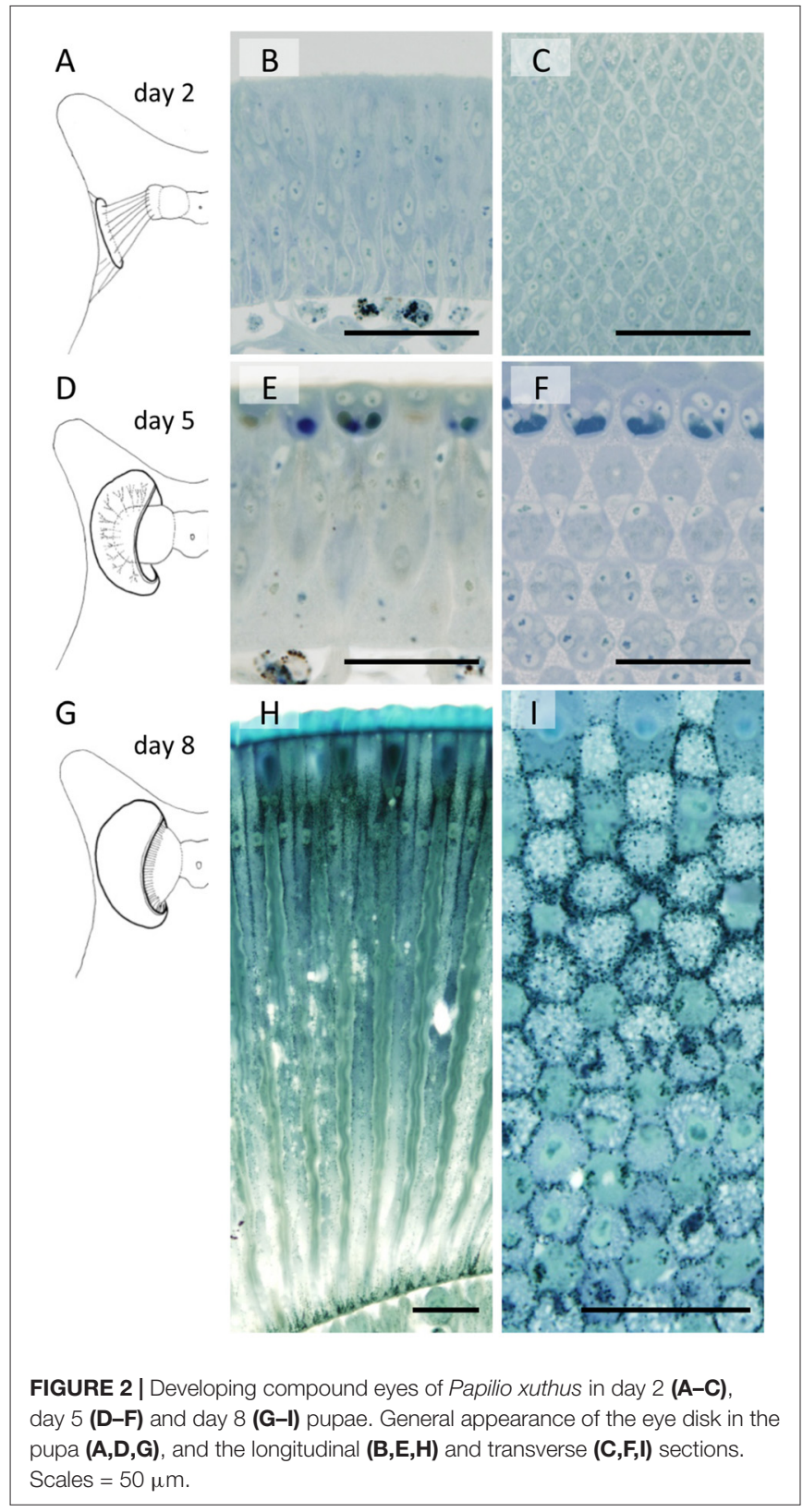

formed in day 5 (Figure 2D) and pigmentation starts on day 8 (Figures 2H,I).

Figure 2 also shows sections of developing eye tissues, cut parallel (middle column) and perpendicular (right column) to the ommatidial optical axis. Because of the curvature of the tissue, the perpendicular sections are actually slightly oblique, showing transverse views of ommatidia at different levels within single sections.

By day 2 we can already identify hexagonally arranged cell groups, corresponding to single ommatidia (Figures $\mathbf{2 A - C}$ ). However, cell types (e.g., photoreceptors or pigment cells) are not clear from the conventional histology at the light microscopic level. On day 5, precursors of crystalline cone cells, photoreceptor cells, and pigment cells can begin to be distinguished according to the positions of the nuclei (Figures 2E,F). The basic
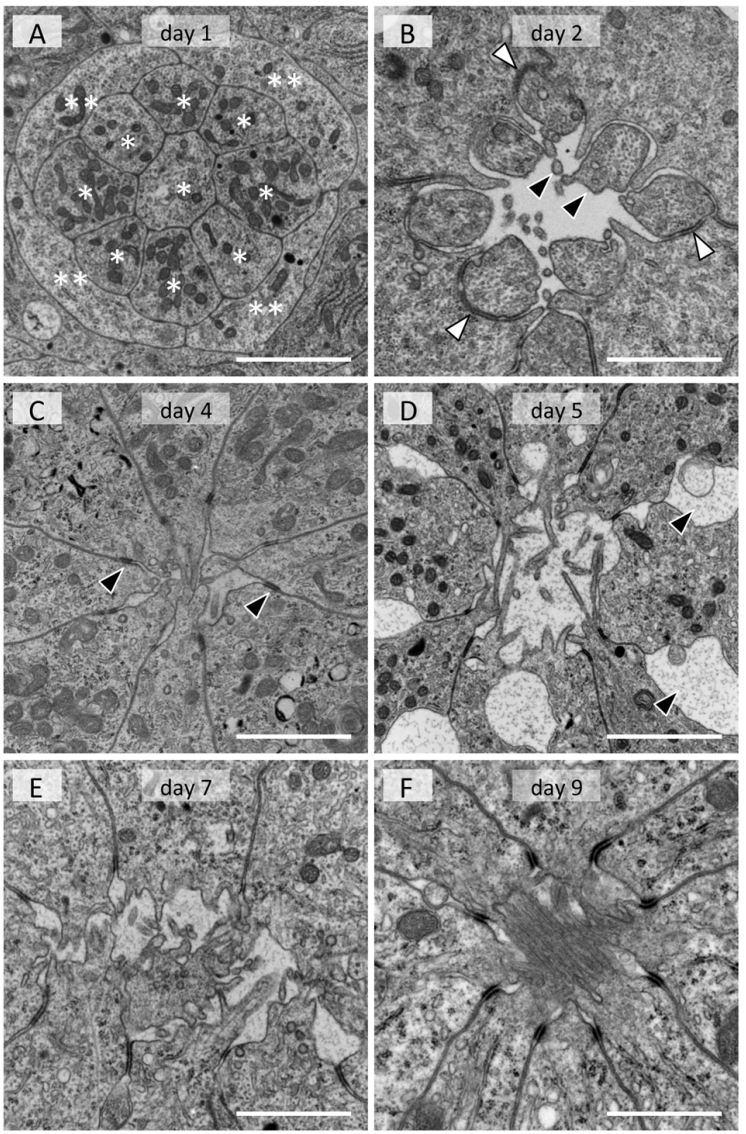

FIGURE 3 | Electron micrographs of developing rhabdom in the eye disk from pupae. (A) Day 1. Eight columnar cells $\left({ }^{*}\right)$ and flat cells $\left({ }^{* *}\right)$ are evident.

(B) Day 2. Membrane of photoreceptor precursors start evaginating (black arrowheads). White arrowheads indicate desmosome-like junctions.

(C) Day 4. Arrowheads indicate interphotoreceptor junctions as in adult ommatidia (see Figure 1B). (D) Day 5. Interphotoreceptor spaces enlarge (arrowheads). (E) Day 7. (F) Day 9. Scales $=2 \mu \mathrm{m}$.

compartments of the ommatidia are all clearly identifiable by day 6 . The length of the ommatidia are about $130 \mu \mathrm{m}$, which increases over the next 3 days, reaching more than $450 \mu \mathrm{m}$ on day 8 with a characteristic two-tiered configuration (Figures 1A, 2G-I). This developmental process is similar to that of Manduca sexta (Monsma and Booker, 1996).

\section{Ultrastructure}

In the adult, photoreceptor cells extend numerous tightly packed microvilli towards the center of the ommatidium, forming a fused rhabdom about $2 \mu \mathrm{m}$ in diameter (Figure 1B). In the day 1 tissue, groups of 13 cells are evident at the electron microscopic level. A group always has a columnar cell in the center, which is surrounded by eight other columnar cells (single asterisks in Figure 3A). Four cells that appear flat in transverse sections wrap around the group of nine columnar cells (double asterisks in Figure 3A). The space between the cell groups is filled with rough ER-rich cells. Presumably, the columnar, flat and rER-rich cells respectively correspond to photoreceptor, cone and pigment cell 
precursors. Therefore we hereafter refer to these precursor cells as photoreceptors, cone cells and pigment cells for simplicity.

On day 2, a cavity appears in the center, surrounded by eight photoreceptor cells (Figure 3B). The cell which was in the center on the previous day (most likely the precursor of the basal photoreceptor R9) moves to the proximal end of the extending ommatidium. The eight remaining photoreceptors start to evaginate irregular processes towards the cavity (black arrowheads in Figure 3B). The cone cells enlarge and interpose between photoreceptor cells, forming belt desmosome-like junctions with the photoreceptor cells in places (white arrowheads in Figure 3B).

The cross sectional area of the photoreceptor cells increases on day 4 (Figure 3C). The cone cell cytoplasm between the photoreceptors disappears and thus the photoreceptor cells come into direct contact, forming belt desmosomes between them as in the adult (arrowheads in Figure 3C). The photoreceptor cells continue to extend processes into the cavity.

The most notable feature of the day 5 tissues is the enlarged inter-photoreceptor spaces (arrowheads in Figure 3D), indicating that the packing of the photoreceptors becomes somewhat loose. The ommatidia extensively elongate from day 6 to 9 , so these two phenomena may be related.

The diameter of the photoreceptor processes in the central cavity is variable on day 7 (Figure 3E), but it becomes about $80 \mathrm{~nm}$, which is equivalent to the adult microvilli, by day 8 . Although the cavity is mostly filled with microvilli by day 9 (Figure 3F), the arrangement of microvilli is still incomplete (see Figure 1B). The rhabdom formation is probably completed shortly after adult eclosion.

\section{Expression and Localization of Opsin mRNAs}

Figure 4 shows the results of RT-PCR of five opsin mRNAs, as well as the mRNAs of Papilio actin. The concentration of the template cDNA is adjusted to $5 \mu \mathrm{g} / \mu \mathrm{l}$, which gives stable amplification results in the actin control (see bottom row). Not all opsin mRNAs appear simultaneously. The mRNAs of PxUV and $\mathrm{PxB}$ are already detectable on day 1 . Those of PxG2 and PxR become detectable on day 2 and day 4, respectively, but the PxG1 mRNA does not appear until day 6 .

We carried out histological in situ hybridization of five opsin mRNAs in the developing eye tissue isolated from pupae of various stages (Figure 5), and could identify signals by in situ hybridization 4 days after the detection of all opsin mRNAs by RT-PCR. This presumably reflects the difference in sensitivity between these two methods.

The right-most column of Figure 5 shows the distribution of opsin mRNAs in the adult, which is summarized in Figure 1D (Arikawa, 2003). The three framed pairs (b/e, c/f, l/q) are adjacent sections labeled with different probes. Solid, broken and dotted circles in these pictures indicate type I, II and III ommatidia, respectively.

On day 3, no signals are detectable. The probes specific to $\mathrm{PxUV}$ and $\mathrm{PxB} \mathrm{mRNAs}$ give adult-like labeling on day 4 (Figures 5B,C,E,F). Three types of ommatidia are evident according to the labeling pattern in Papilio R1 (pR1) and

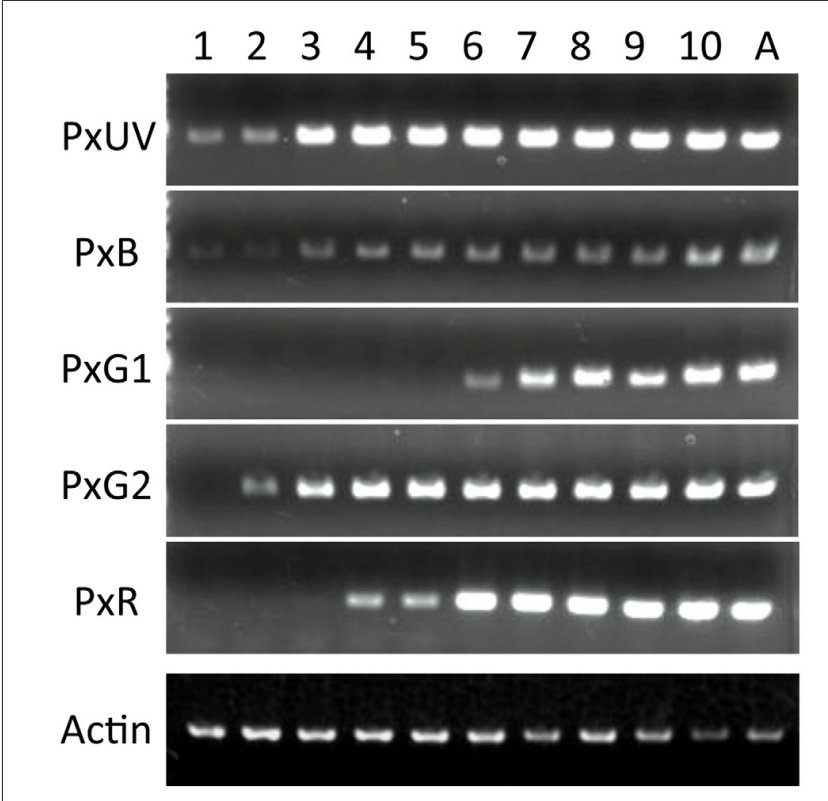

FIGURE 4 | Expression analysis of five visual pigment opsins by RT-PCR. Numbers on the top indicate days after pupation, and A for adult. As a control, the actin cDNA is amplified.

pR2: PxUV-PxB (I in Figure 5B), PxUV-PxUV (II), and $\mathrm{PxB}-\mathrm{PxB}$ (III). This indicates that the fates of the $\mathrm{pR} 1$ and pR2 photoreceptors are already determined by day 4 .

The three mRNAs encoding long-wavelength opsins appear later. The expression of the PxG1 mRNA is detected only after day 9 in the pR3 and pR4 photoreceptors of all ommatidia (Figures 5G,H), as in the adult (Figure 5I). As seen in Figure 5L, these photoreceptors coexpress PxG2 mRNA (Kitamoto et al., 1998). PxG2 mRNA is first detected on day 5 in the pR3,4 distal photoreceptors and pR5-8 proximal photoreceptors of all ommatidia (6 arrowheads in Figure 5K). Because the ommatidia are still short and the tiered-configuration is not yet formed on day 5 , all six of these photoreceptors are visible in a single section (Figure 5K). Intriguingly, the PxG2 labeling in pR5-8 proximal receptors disappears on day 7 in a number of ommatidia, while the labeling in pR3 and pR4 remains (solid and broken circles in Figure 5L). These pR5-8 photoreceptors in some ommatidia instead express the PxR mRNA (solid circle in Figure 5Q); these correspond to type I ommatidia in the adult. In some other ommatidia, the cells were labeled faintly with the PxR probe (broken circle in Figure 5Q); these are type II ommatidia (Figure $5 \mathbf{N}$ ), whose pR5-8 photoreceptors are the broad-band receptors coexpressing PxG2 and PxR visual pigments (Arikawa et al., 2005).

\section{DISCUSSION}

\section{Comparison with Drosophila Eye Development}

Development of insect compound eyes has been extensively studied in Drosophila melanogaster (Ready et al., 1976; 


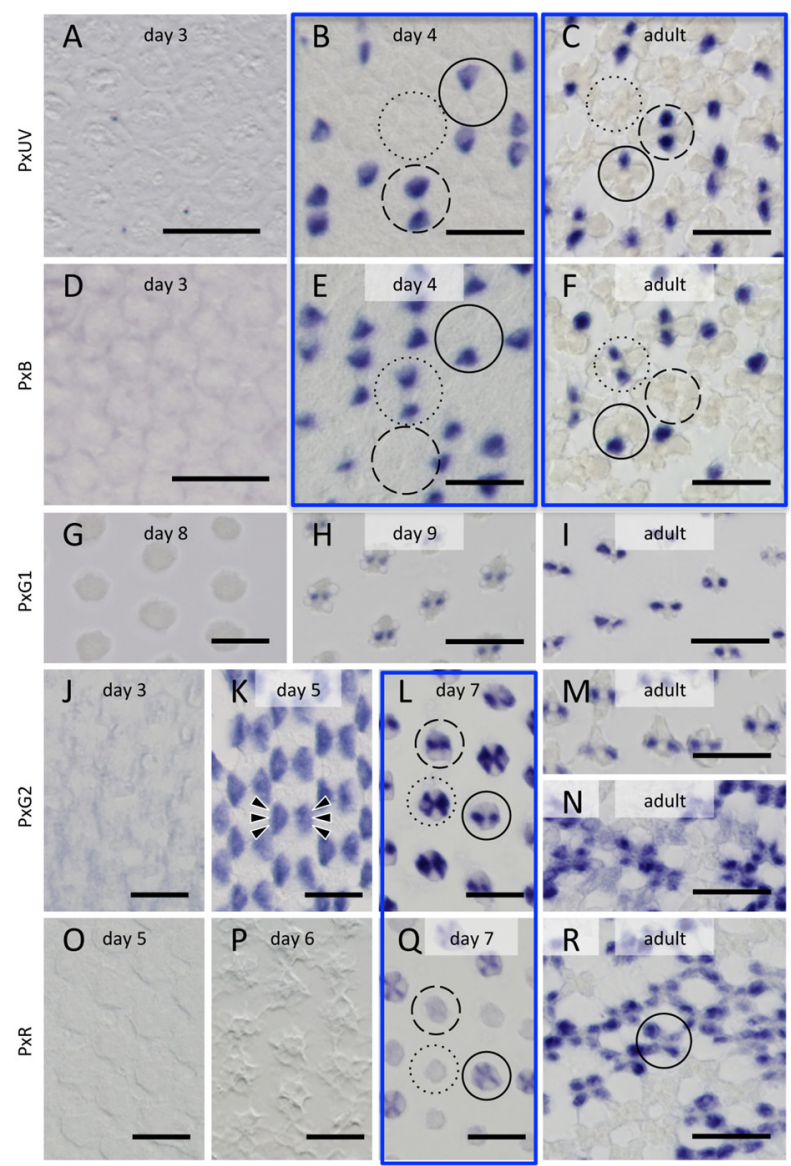

FIGURE 5 | Histological in situ hybridization of five opsin mRNAs in the developing and adult compound eyes of Papilio xuthus. (A-C) PxUV. (D-F) PxB. (G-I) PxG1. (J-N) PxG2. (O-R) PxR. Developmental stages are indicated in each pictures. Three framed pairs are from adjacent sections labeled with different probes. Solid, broken and dotted circles indicate type I, II and III ommatidia. Arrowheads in (K) indicate six cells labeled.

Scales $=20 \mu \mathrm{m}$.

Cagan and Ready, 1989). In Drosophila, the pupal stage lasts for $160 \mathrm{~h}$ at $20^{\circ} \mathrm{C}$ (Cagan and Ready, 1989) and the fate of ommatidia is determined early in the pupal stage (Michael Perry, personal communication; Wernet et al., 2006). The first mRNA encoding a visual pigment opsin, Rh1, then appears at $78 \%$ of the way through pupal development ( $\mathrm{pd}, 78 \%$ ), which is followed by the expression of mRNAs encoding Rh3, 4 and 5 at $80 \%$ pd, and Rh6 at $82 \%$ pd (Earl and Britt, 2006). Expression of the respective opsin proteins follows the same course with a slight (1\%-2\% pd) delay (Earl and Britt, 2006).

Figure 6 summarizes the process of pupal development of Papilio in terms of the expression of opsin mRNAs. Ommatidial fate determination is completed in phase 1 as in Drosophila (Perry et al., 2016). Interestingly, in Papilio opsin mRNAs are already detectable at $10 \%$ pd via RT-PCR. It requires four more days before mRNA becomes detectable in histological in situ hybridization, presumably because RT-PCR is a more sensitive technique. We tried to localize opsin proteins by immunohistochemistry using specific antibodies against PxUV, $\mathrm{PxB}$ and $\mathrm{PxG} 2$ in the eye disks taken from pupae of $\sim 60 \%$ pd. Although those antibodies successfully label subsets of photoreceptors in adults (Perry et al., 2016), we could not detect any signs of labeling in the pupal eye disks. This strongly indicates that opsin proteins are not synchronously expressed with their mRNAs, unlike in Drosophila (Kumar and Ready, 1995).

Rhabdomere formation happens much earlier in Drosophila than in Papilio. Drosophila photoreceptors already exhibit short but organized arrays of processes on the apical side by $55 \%$ $\mathrm{pd}$. The developing rhabdomeral structures start to separate at $73 \% \mathrm{pd}$, eventually forming the open rhabdomere configuration by $90 \%$ pd (Kumar and Ready, 1995). On the other hand, the rhabdomeral microvilli of Papilio photoreceptors are quite immature even at $70 \% \mathrm{pd}$. Formation of a clear border between the rhabdomere and the photoreceptor cell body, i.e., the structure of the microvillar base, starts very late at around $90 \% \mathrm{pd}$ (Figure 3). Opsins are essential for making the microvillar base structure in Drosophila (Kumar and Ready, 1995). Assuming a similar structural function of Papilio opsins in developing photoreceptors, the late translation of mRNAs into opsin proteins is likely related to this phenomenon.

\section{Temporal Coexpression of Opsin mRNAs}

Results from in situ hybridization experiments are summarized in Figure 7. The Papilio R9 (pR9) photoreceptor has been specified by this point (see Figure $\mathbf{3 A}$ ), as shown via immunohistochemistry using whole-mount preparations (Perry et al., 2016). However, we could not identify these cells in the sections cut for in situ hybridization. Either they were located outside the sections, the mRNA concentration was too low to be detected, or mRNA expression began even later in pR9.

The complimentary expression of PxUV and PxB mRNAs in $\mathrm{pR} 1$ and pR2 precursors in day 4 indicates that the ommatidial fate specification is complete by then (Figures $5 \mathrm{~B}, \mathbf{E}$ ). The other photoreceptors, pR3-8, are all long-wavelength sensitive. Quite surprisingly, L opsin expression is uniform at the beginning: PxG2 mRNA is expressed in all pR3-8 cells in all ommatidia on day 5 (Figure $5 \mathbf{K}$ ). This uniform pattern is similar to that observed in other butterfly species (Wakakuwa et al., 2004; Sison-Mangus et al., 2006; Uchiyama et al., 2013; McCulloch et al., 2016) and honeybees (Wakakuwa et al., 2005), which have only one L opsin in their genome. This simple uniform pattern persists into adulthood only in type III ommatidia in Papilio.

The Papilio pR3-8 photoreceptors are both structurally and spectrally heterogeneous. Structurally, pR3 and pR4 contribute to the distal tier of the two-tiered rhabdom, while pR5-8 form the proximal tier. The distal pR3 and pR4 photoreceptors are green sensitive in all ommatidia, suggesting they function in motion and shape vision: they form the basis for a quasiindependent achromatic system for detecting motion (Takemura and Arikawa, 2006; Stewart et al., 2015). According to Friedrich et al. (2011), the butterfly's pR3/4 pair is homologous to fly's dR2/5 pair, which differentiates first among dR1-6 immediately 


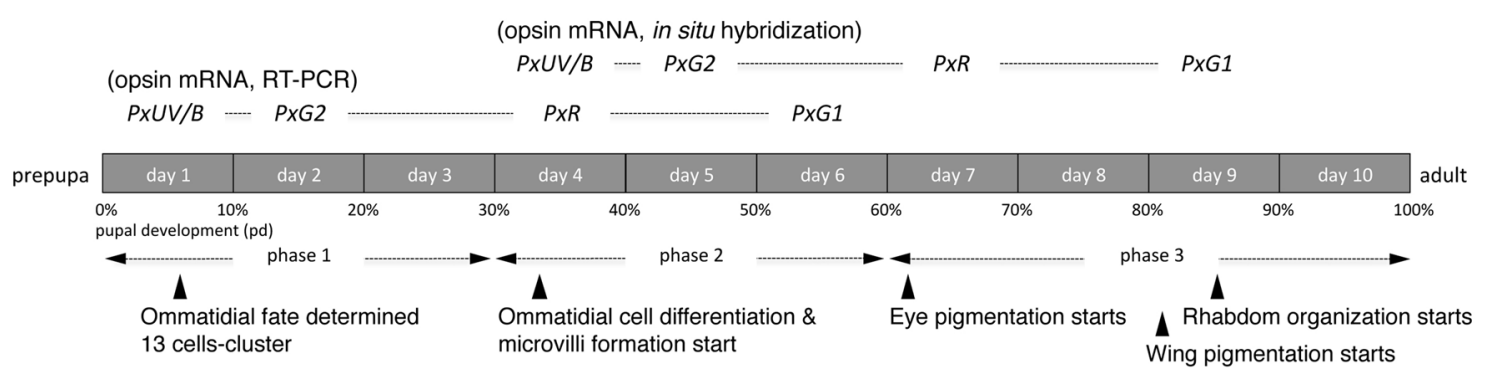

FIGURE 6 | Development of the Papilio pupal eye disk.

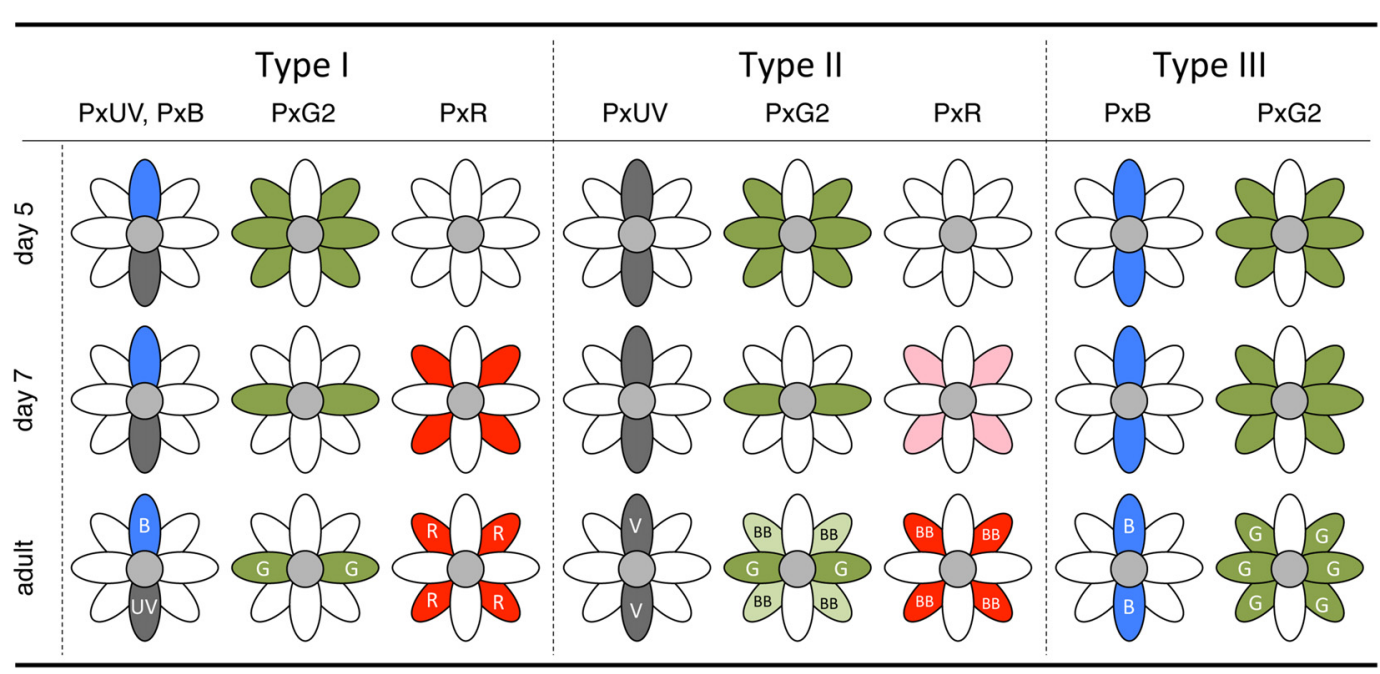

FIGURE 7 | Summary diagram of the in situ hybridization results of day 5 and day 7 pupae with the final state in the adult.

after dR8 differentiation (pR9 in the case of Papilio). This early differentiation may be linked to the structural and functional distinction of pR3 and pR4 in Papilio. The spectral heterogeneity of the pR5-8 proximal photoreceptors, which are involved in color vision (Arikawa, 2003; Koshitaka et al., 2008), is apparent by day 7. While labeling with the PxG2 probe in pR3 and pR4 remains constant, the precursors of pR5-8 lose PxG2 mRNA expression in type I and II ommatidia. These photoreceptors then express PxR mRNA strongly in type I and weakly in type II. Expression of PxG2 mRNA recovers to some extent in pR5-8 cells of type II ommatidia in the adult, which are broad-band receptors coexpressing PxG2 and PxR (Figure 1).

Because we failed to detect opsin proteins in pupal eye discs, we conclude that opsin proteins are not expressed until rhabdom formation enters its final stage on day 9: the PxG2 mRNA that is expressed transiently in pR5-8 of type I and II ommatidium on day 5 is, therefore, never translated. This correlates with the delayed rhabdom formation in Papilio, which happens only after the necessary opsin proteins are ready. Rhabdom formation may start earlier in the eyes of species with simpler spectral organization, which express only three basic opsins.
Among lepidopterans, one such example is the silk moth Bombyx mori (Mita et al., 2004; Xia et al., 2004; Briscoe, 2008), where developing rhabdoms are clearly recognizable at $\sim 50 \% \mathrm{pd}$. These developing eyes are even able to produce receptor potentials in response to light stimulation (Eguchi et al., 1962), which requires visual pigments.

\section{Perspectives from a Comparative Point of View}

The observed temporal and spatial patterns of expression of opsin mRNAs in Papilio xuthus may have resulted from the evolution of additional opsins. Chen et al. (2016) identified opsins of ten species from four tribes in the family Papilionidae, and found that their L opsins cluster into three clades: L1, L2, and L3. These evolutionary relationships (Figure 2 of Chen et al., 2016) suggest that the ancestral L opsin was duplicated, with one opsin becoming L2 (in this case PxG2 with the peak absorption $\left(\lambda_{\max }\right)$ at $\left.515 \mathrm{~nm}\right)$ and a second opsin that later duplicated to produce L1 (PxG1 in P. xuthus, $\lambda_{\max }=545 \mathrm{~nm}$ ) and L3 (PxR in P. xuthus, $\lambda_{\max }=575 \mathrm{~nm}$ ) (Kinoshita et al., 2006). Uniform expression of the PxG2 mRNA in pR3-8 on 
day 5 (Figure 6) is consistent with the ancestral nature of PxG2. L1 opsins are found only in the tribe Papilionini, and at least in P. xuthus and P. glaucus L1 is always coexpressed with L2 in $\mathrm{pR} 3$ and pR4 photoreceptors in the ventral retina (Kitamoto et al., 1998; Briscoe, 2008). Perhaps neofunctionalization of PxG1 is not sufficient to create photoreceptors with distinct spectral sensitivity. On the other hand, the newly evolved red-absorbing PxR clearly contributes to produce red sensitive photoreceptors (Arikawa et al., 1999). The expression of PxR mRNA is always preceded by the expression of PxG2 mRNA; perhaps this order of expression simply reflects ancestral regulation, or perhaps PxR expression is itself regulated by earlier PxG2 expression.

Mechanisms that might control the temporal and spatial expression of mRNAs and the observed post-transcriptional delay in protein production remains to be studied in detail. The opsin expression pattern and the photoreceptor spectral sensitivities observed in Papilio (Figure 1D) are not conserved even among butterfly species. Because of this variability, comparative studies among butterflies may be a useful approach toward understanding the general principles that underlie the spatial and temporal control of opsin expression.

In the family Pieridae, $\mathrm{M}$ opsin duplication is common (Ogawa et al., 2012). M opsins are restricted to the pR1 and pR2, as in Papilio. The expression pattern is however slightly more complex and variable. The cabbage white Pieris rapae (Pieridae, Lepidoptera) has one S (PrUV) and two M (PrV and $\operatorname{PrB}$ ) opsins, which are expressed in three combinations, UV/B, $\mathrm{UV} / \mathrm{UV}$ and V/V. A similar case is found in the red postman Heliconius erato (Nymphalidae, Lepidoptera), where S opsin is duplicated (Briscoe et al., 2010; McCulloch et al., 2016). The expression of the $\mathrm{S}$ and $\mathrm{M}$ opsins must be under the control of Spineless, but the involvement of a third opsin requires at least one additional mechanism. For example, an additional step is required in Pieris to explain how Spineless ON-ON results in $\mathrm{V} / \mathrm{V}$, rather than $\mathrm{B} / \mathrm{B}$ as in Papilio (Figure 1D). It cannot be that $\mathrm{V}$ simply takes the place of $\mathrm{B}$, as this does not explain the UV/B

\section{REFERENCES}

Arikawa, K. (2003). Spectral organization of the eye of a butterfly, Papilio. J. Comp. Physiol. A 189, 791-800. doi: 10.1007/s00359-003-0454-7

Arikawa, K., Mizuno, S., Kinoshita, M., and Stavenga, D. G. (2003). Coexpression of two visual pigments in a photoreceptor causes an abnormally broad spectral sensitivity in the eye of a butterfly, Papilio xuthus. J. Neurosci. 23, 4527-4532.

Arikawa, K., Scholten, D. G. W., Kinoshita, M., and Stavenga, D. G. (1999). Tuning of photoreceptor spectral sensitivities by red and yellow pigments in the butterfly Papilio xuthus. Zoolog. Sci. 16, 17-24. doi: 10.2108/zsj.16.17

Arikawa, K., Wakakuwa, M., Qiu, X., Kurasawa, M., and Stavenga, D. G. (2005). Sexual dimorphism of short-wavelength photoreceptors in the Small White butterfly, Pieris rapae crucivora. J. Neurosci. 25, 5935-5942. doi: 10.1523/ JNEUROSCI.1364-05.2005

Briscoe, A. D. (1999). Intron splice sites of Papilio glaucus PglRh3 corroborate insect opsin phylogeny. Gene 230, 101-109. doi: 10.1016/s0378-1119(99) 00027-x

Briscoe, A. D. (2008). Reconstructing the ancestral butterfly eye: focus on the opsins. J. Exp. Biol. 211, 1805-1813. doi: 10.1242/jeb.013045 pattern in the ON-OFF case. The situation is more complex still in the eastern clouded yellow, Colias erate, which has one $\mathrm{S}(\mathrm{CeUV})$ and three $\mathrm{M}(\mathrm{CeB}, \mathrm{CeV} 1$ and $\mathrm{CeV} 2)$ opsins (Ogawa et al., 2012). CeV1 is always coexpressed with V2, and a subset of V1-V2 coexpressing cells has $\mathrm{CeB}$ as the third opsin; $\mathrm{CeB}$ is not expressed anywhere else. This pattern suggests that these $\mathrm{M}$ opsins are in the process of neofunctionalization, possibly like PxL1 in Papilio.

The most complex case ever reported is the ruddy copper, Lycaena rubidus (Lycaenidae, Lepidoptera), which has one $\mathrm{S}$ (UV) and two $\mathrm{M}$ (B1 and B2) opsins (Sison-Mangus et al., 2006). The authors report six types of ommatidia in Lycaena rubidus with all possible pairwise combinations of three opsins (UV/UV, UV/B1, UV/B2, B1/B1, B1/B2, B2/B2) in $\mathrm{pR} 1$ and $\mathrm{pR} 2$, while all other species so far studied have only three types. Moreover, B2 opsin is coexpressed in pR3-8 long-wavelength receptors with the $\mathrm{L}$ opsin in the dorsal region of the eye, but only in females. This is peculiar because the B2 opsin and L opsin are genetically distant, and here they break the "boundary" of the short-wavelength $(\mathrm{pR} 1,2)$ and long-wavelength (pR5-8) photoreceptors within the ommatidia. The sex-specific "transboundary" expression of an M opsin in L receptors could be of particular interest to reveal the evolution of color vision in these flower-visiting insects.

\section{AUTHOR CONTRIBUTIONS}

KA, TI, MW and MK jointly conceived the project and designed the experiments. TI, MW and MK performed the experiments. KA and MK supervised the project. KA wrote the article. All authors discussed the results and manuscript.

\section{ACKNOWLEDGMENTS}

We thank Drs. Michael Perry and Finlay Stewart for extensive discussion and editing the English. This work was supported by the KAKENHI of the Japan Society for the Promotion of Science (JSPS; \#26650117, \#26251036) to KA.
Briscoe, A. D., Bybee, S. M., Bernard, G. D., Yuan, F., Sison-Mangus, M. P., Reed, R. D., et al. (2010). Positive selection of a duplicated UV-sensitive visual pigment coincides with wing pigment evolution in Heliconius butterflies. Proc Natl. Acad. Sci. U S A 107, 3628-3633. doi: 10.1073/pnas.09100 85107

Cagan, R. L., and Ready, D. F. (1989). The emergence of order in the Drosophila pupal retina. Dev. Biol. 136, 346-362. doi: 10.1016/0012-1606(89) 90261-3

Chang, B. S., Ayers, D., Smith, W. C., and Pierce, N. E. (1996). Cloning of the gene encoding honeybee long-wavelength rhodopsin: a new class of insect visual pigments. Gene 173, 215-219. doi: 10.1016/0378-1119(96)00165-5

Chen, P.-J., Awata, H., Matsushita, A., Yang, E.-C., and Arikawa, K. (2016). Extreme spectral richness in the eye of the common bluebottle butterfly, Graphium sarpedon. Front. Ecol. Evol. 4:18. doi: 10.3389/fevo.2016.00018

Chou, W. H., Huber, A., Bentrop, J., Schulz, S., Schwab, K., Chadwell, L. V., et al. (1999). Patterning of the R7 and R8 photoreceptor cells of Drosophila: evidence for induced and default cell-fate specification. Development 126, 607-616.

Cook, T., Pichaud, F., Sonneville, R., Papatsenko, D., and Desplan, C. (2003). Distinction between color photoreceptor cell fates is controlled by Prospero in Drosophila. Dev. Cell 4, 853-864. doi: 10.1016/s1534-5807(03)00156-4 
Dalton, B. E., Loew, E. R., Cronin, T. W., and Carleton, K. L. (2014). Spectral tuning by opsin coexpression in retinal regions that view different parts of the visual field. Proc. Biol. Sci. 281:20141980. doi: 10.1098/rspb.2014.1980

Earl, J. B., and Britt, S. G. (2006). Expression of Drosophila rhodopsins during photoreceptor cell differentiation: insights into R7 and R8 cell subtype commitment. Gene Expr. Patterns 6, 687-694. doi: 10.1016/j.modgep.2006. 01.003

Eguchi, E., Naka, K. I., and Kuwabara, M. (1962). The development of the rhabdom and the appearance of the electrical response in the insect eye. J. Gen. Physiol. 46, 143-157. doi: 10.1085/jgp.46.1.143

Franceschini, N., Kirschfeld, K., and Minke, B. (1981). Fluorescence of photoreceptor cells observed in vivo. Science 213, 1264-1267. doi: 10.1126/science.7268434

Friedrich, M., Wood, E. J., and Wu, M. (2011). Developmental evolution of the insect retina: insights from standardized numbering of homologous photoreceptors. J. Exp. Zool. B Mol. Dev. Evol. 316, 484-499. doi: 10.1002/jez.b. 21424

Hu, X., Whaley, M. A., Stein, M. M., Mitchell, B. E., and O’Tousa, J. E. (2011). Coexpression of spectrally distinct rhodopsins in Aedes aegypti R7 photoreceptors. PLoS One 6:e23121. doi: 10.1371/journal.pone.0023121

Kinoshita, M., Kurihara, D., Tsutaya, A., and Arikawa, K. (2006). Blue and doublepeaked green receptors depend on ommatidial type in the eye of the Japanese yellow swallowtail Papilio xuthus. Zoolog. Sci. 23, 199-204. doi: 10.2108/zsj. 23.199

Kitamoto, J., Ozaki, K., and Arikawa, K. (2000). Ultraviolet and violet receptors express identical mRNA encoding an ultraviolet-absorbing opsin: identification and histological localization of two mRNAs encoding shortwavelength-absorbing opsins in the retina of the butterfly Papilio xuthus. J. Exp. Biol. 203, 2887-2894.

Kitamoto, J., Sakamoto, K., Ozaki, K., Mishina, Y., and Arikawa, K. (1998). Two visual pigments in a single photoreceptor cell: identification and histological localization of three mRNAs encoding visual pigment opsins in the retina of the butterfly Papilio xuthus. J. Exp. Biol. 201, 1255-1261.

Koshitaka, H., Kinoshita, M., Vorobyev, M., and Arikawa, K. (2008). Tetrachromacy in a butterfly that has eight varieties of spectral receptors. Proc. Biol. Sci. 275, 947-954. doi: 10.1098/rspb.2007.1614

Kumar, J. P., and Ready, R. F. (1995). Rhodopsin plays an essential structural role in Drosophila photoreceptor development. Development 121, 4359-4370.

Makino, C. L., and Dodd, R. L. (1996). Multiple visual pigments in a photoreceptor of the salamander retina. J. Gen. Physiol. 108, 27-34. doi: 10.1085/jgp.108.1.27

Mazzoni, E. O., Celik, A., Wernet, M. F., Vasiliauskas, D., Johnston, R. J., Cook, T. A., et al. (2008). Iroquois complex genes induce co-expression of rhodopsins in Drosophila. PLoS Biol. 6:e97. doi: 10.1371/journal.pbio.00 60097

McCulloch, K. J., Osorio, D., and Briscoe, A. D. (2016). Sexual dimorphism in the compound eye of Heliconius erato: a nymphalid butterfly with at least five spectral classes of photoreceptor. J. Exp. Biol. 219, 2377-2387. doi: 10.1242/jeb. 136523

McCulloch, K. J., Yuan, F., Zhen, Y., Aardema, M. L., Smith, G., LlorenteBousquets, J., et al. (2017). Sexual dimorphism and retinal mosaic diversification following the evolution of a violet receptor in butterflies. Mol. Biol. Evol. 34, 2271-2284. doi: 10.1093/molbev/msx163

Mita, K., Kasahara, M., Sasaki, S., Nagayasu, Y., Yamada, T., Kanamori, H., et al. (2004). The genome sequence of silkworm, Bombyx mori. DNA Res. 11, 27-35. doi: 10.1093/dnares/11.1.27

Monsma, S. A., and Booker, R. (1996). Genesis of the adult retina and outer optic lobes of the moth, Manduca sexta. 1. Patterns of proliferation and cell death. J. Comp. Neurol. 367, 10-20. doi: 10.1002/(SICI)10969861(19960325)367:1<10::AID-CNE2>3.0.CO;2-M

Ogawa, Y., Awata, H., Wakakuwa, M., Kinoshita, M., Stavenga, D. G., and Arikawa, K. (2012). Coexpression of three middle wavelength-absorbing visual pigments in sexually dimorphic photoreceptors of the butterfly Colias erate. J. Comp. Physiol. A 198, 857-867. doi: 10.1007/s00359-012-0756-8

Parry, J. W., and Bowmaker, J. K. (2002). Visual pigment coexpression in Guinea pig cones: a microspectrophotometric study. Invest. Ophthalmol. Vis. Sci. 43, $1662-1665$.
Perry, M., Kinoshita, M., Saldi, G., Huo, L., Arikawa, K., and Desplan, C. (2016). Molecular logic behind the three-way stochastic choices that expand butterfly colour vision. Nature 535, 280-284. doi: 10.1038/nature18616

Rajkumar, P., Rollmann, S. M., Cook, T. A., and Layne, J. E. (2010). Molecular evidence for color discrimination in the Atlantic sand fiddler crab, Uca pugilator. J. Exp. Biol. 213, 4240-4248. doi: 10.1242/jeb.051011

Ready, D. F., Hanson, T. E., and Benzer, S. (1976). Development of the Drosophila retina, a neurocrystalline lattice. Dev. Biol. 53, 217-240. doi: 10.1016/00121606(76)90225-6

Röhlich, P., van Veen, T., and Szél, A. (1994). Two different visual pigments in one retinal cone cell. Neuron 13, 1159-1166. doi: 10.1016/0896-6273(94)90053-1

Schmeling, F., Wakakuwa, M., Tegtmeier, J., Kinoshita, M., Bockhorst, T., Arikawa, K., et al. (2014). Opsin expression, physiological characterization and identification of photoreceptor cells in the dorsal rim area and main retina of the desert locust, Schistocerca gregaria. J. Exp. Biol. 217, 3557-3568. doi: 10.1242/jeb.108514

Sison-Mangus, M. P., Bernard, G. D., Lampel, J., and Briscoe, A. D. (2006). Beauty in the eye of the beholder: the two blue opsins of lycaenid butterflies and the opsin gene-driven evolution of sexually dimorphic eyes. J. Exp. Biol. 209, 3079-3090. doi: 10.1242/jeb.02360

Stewart, F. J., Kinoshita, M., and Arikawa, K. (2015). The butterfly Papilio xuthus detects visual motion using chromatic contrast. Biol. Lett. 11:20150687. doi: $10.1098 /$ rsbl.2015.0687

Takemura, S. Y., and Arikawa, K. (2006). Ommatidial type-specific interphotoreceptor connections in the lamina of the swallowtail butterfly, Papilio xuthus. J. Comp. Neurol. 494, 663-672. doi: 10.1002/cne.20830

Townson, S. M., Chang, B. S. W., Salcedo, E., Chadwell, L. V., Pierce, N. E., and Britt, S. G. (1998). Honeybee blue-and ultraviolet-sensitive opsins: cloning, heterologous expression in Drosophila, and physiological characterization. J. Neurosci. 18, 2412-2422.

Uchiyama, H., Awata, H., Kinoshita, M., and Arikawa, K. (2013). Rough eyes of the northeast-Asian wood white Leptidea amurensis. J. Exp. Biol. 216, 3414-3421. doi: 10.1242/jeb.089169

von Helversen, O. (1972). Zur spektralen Unterschiedsempfindlichkeit der Honigbiene. J. Comp. Physiol. 80, 439-472. doi: 10.1007/bf00696438

Wakakuwa, M., Kurasawa, M., Giurfa, M., and Arikawa, K. (2005). Spectral heterogeneity of honeybee ommatidia. Naturwissenschaften 92, 464-467. doi: 10.1007/s00114-005-0018-5

Wakakuwa, M., Stavenga, D. G., Kurasawa, M., and Arikawa, K. (2004). A unique visual pigment expressed in green, red and deep-red receptors in the eye of the Small White butterfly, Pieris rapae crucivora. J. Exp. Biol. 207, 2803-2810. doi: 10.1242/jeb.01078

Wakakuwa, M., Stewart, F., Matsumoto, Y., Matsunaga, S., and Arikawa, K. (2014). Physiological basis of phototaxis to near infrared light in Nephotettix cincticeps. J. Comp. Physiol. A 200, 527-536. doi: 10.1007/s00359-014-0892-4

Wernet, M. F., Mazzoni, E. O., Çelik, A., Duncan, D. M., Duncan, I., and Desplan, C. (2006). Stochastic spineless expression creates the retinal mosaic for colour vision. Nature 440, 174-180. doi: 10.1038/nature04615

Wernet, M. F., Perry, M. W., and Desplan, C. (2015). The evolutionary diversity of insect retinal mosaics: common design principles and emerging molecular logic. Trends Genet. 31, 316-328. doi: 10.1016/j.tig.2015.04.006

Xia, Q., Zhou, Z., Lu, C., Cheng, D., Dai, F., Li, B., et al. (2004). A draft sequence for the genome of the domesticated silkworm (Bombyx mori). Science 306, 1937-1940. doi: 10.1126/science.1102210

Conflict of Interest Statement: The authors declare that the research was conducted in the absence of any commercial or financial relationships that could be construed as a potential conflict of interest.

Copyright (c) 2017 Arikawa, Iwanaga, Wakakuwa and Kinoshita. This is an open-access article distributed under the terms of the Creative Commons Attribution License (CC BY). The use, distribution or reproduction in other forums is permitted, provided the original author(s) or licensor are credited and that the original publication in this journal is cited, in accordance with accepted academic practice. No use, distribution or reproduction is permitted which does not comply with these terms. 